\title{
Impurity-induced turbulence suppression and reduced transport in the DIII-D tokamak*
}

\author{
G. R. McKee, ${ }^{\dagger, a), b)}$ M. Murakami, ${ }^{c}$ J. A. Boedo, ${ }^{d)}$ N. H. Brooks, K. H. Burrell, \\ D. R. Ernst, ${ }^{\text {e) }}$ R. J. Fonck, ${ }^{\text {a) }}$ G. L. Jackson, M. Jakubowski, ${ }^{\text {a) }}$ R. J. La Haye, \\ A. M. Messiaen, ${ }^{f)}$ J. Ongena, ${ }^{f)}$ C. L. Rettig, $\left.{ }^{g}\right)$ B. W. Rice, ${ }^{\text {h) }}$ C. Rost, ${ }^{i)}$ G. M. Staebler, \\ R. D. Sydora, ${ }^{\mathrm{j})}$ D. M. Thomas, B. Unterberg, ${ }^{f)}$ M. R. Wade, ${ }^{\mathrm{C})}$ and W. P. West \\ General Atomics, P.O. Box 85608, San Diego, California 92186-5608
}

(Received 15 November 1999; accepted 14 January 2000)

\begin{abstract}
Long wavelength turbulence as well as heat and momentum transport are significantly reduced in the DIII-D tokamak [Plasma Physics and Controlled Nuclear Fusion Research (International Atomic Energy Agency, Vienna, 1987), Vol. I, p. 159] as a result of neon seeding of a low confinement mode negative central shear discharge. Correspondingly, the energy confinement time increases by up to $80 \%$. Fully saturated turbulence measurements near $\rho=0.7(\rho=r / a)$ in the wave number range $0.1 \leqslant k_{\perp} \rho_{s} \leqslant 0.6$, obtained with beam emission spectroscopy, exhibit a significant reduction of fluctuation power after neon injection. Fluctuation measurements obtained with far infrared scattering also show a reduction of turbulence in the core, while the Langmuir probe array measures reduced particle flux in the edge and scrape-off layer. Gyrokinetic linear stability simulations of these plasmas are qualitatively consistent, showing a reduction in the growth rate of ion temperature gradient driven modes for $0<k_{\perp} \rho_{s} \leqslant 1.4$, and nonlinear gyrokinetic simulations show a reduced saturated density fluctuation amplitude. The measured $\omega_{E \times B}$ shearing rate increased at $\rho=0.7$, suggesting that impurity-induced growth rate reduction is acting synergistically with $\omega_{E \times B}$ shear to decrease turbulence and reduce anomalous transport. (C) 2000 American Institute of Physics. [S1070-664X(00)92805-2]
\end{abstract}

\section{INTRODUCTION}

Optimizing the confinement of energy and fuel ions within a magnetically confined plasma is a central challenge to the realization of fusion as a viable energy source. Transport of particles and energy across the confining and stabilizing magnetic field has long been observed experimentally to be far larger than the neoclassical transport predicted by simple binary collisions in realistic magnetic geometries. This anomalously large transport appears to result from turbulence arising from density and temperature gradients and the associated fluctuations in such quantities as density, electrostatic potential, temperature, and magnetic fields. ${ }^{1}$

It has been experimentally demonstrated on several tokamaks that injecting controlled quantities of impurities into

*Paper BI2 6 Bull. Am. Phys. Soc. 44, 22 (1999).

'Invited speaker.

a) Also with: University of Wisconsin-Madison, Madison, Wisconsin.

${ }^{b)}$ Present address: General Atomics, San Diego, California, Electronic mail: mckee@ fusion.gat.com

c) Also with: Oak Ridge National Laboratory, Oak Ridge, Tennessee.

d) Also with: University of California-San Diego, La Jolla, California.

e) Also with: Princeton Plasma Physics Laboratory, Princeton University, Princeton, New Jersey.

f) Also with: Forschungszentrum, Jülich, Germany.

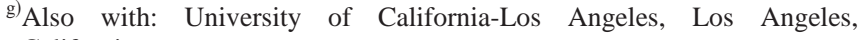
California.

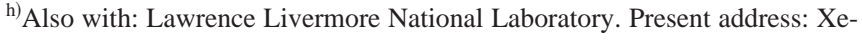
nogen, Alameda, California.

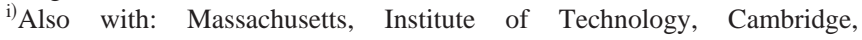
Massachusetts.

${ }^{j}$ Also with: University of Alberta, Edmonton, Alberta, Canada. a plasma discharge results in a significant improvement in the global energy confinement. Electron and ion thermal diffusivities across much of the plasma are dramatically reduced. This behavior was first observed on the Impurity Studies eXperiment-B tokamak $(Z \text { mode })^{2}$ and has been demonstrated more recently on the Tokamak EXperiment for Technology Oriented Research (TEXTOR-94) (RI mode), ${ }^{3}$ Tokamak Fusion Test Reactor (TFTR), ${ }^{4,5}$ and DIII-D tokamaks. ${ }^{6,7}$ These impurity-injected discharges are characterized by high confinement ( $H$ mode)-like energy confinement, reduced transport, higher fusion reactivity, reduced recycling, a highly radiative low confinement $(L)$ mode edge, and reduced intrinsic impurities. On DIII-D, these experiments were extended to a diverted magnetic geometry, and detailed fluctuation measurements of turbulence suppression were obtained and correlated with the reduced transport. The Axi Symmetric Divertor EXperiment Upgrade tokamak has achieved completely detached high-confinement modes by injecting regulated quantities of neon into $H$-mode plasmas with edge localized modes without suffering any loss of energy confinement. ${ }^{8}$

An understanding of one of the physical mechanisms that gives rise to these impurity-induced global discharge improvements is beginning to emerge. It appears that plasma turbulence is partially stabilized and that the $E \times B$ shearing rate subsequently increases as a result of the impurity injection. Probe measurements in the edge region of TEXTOR-94 showed a reduction in turbulent-driven particle flux in this region. ${ }^{9}$ Fluctuation measurements obtained in the core and 


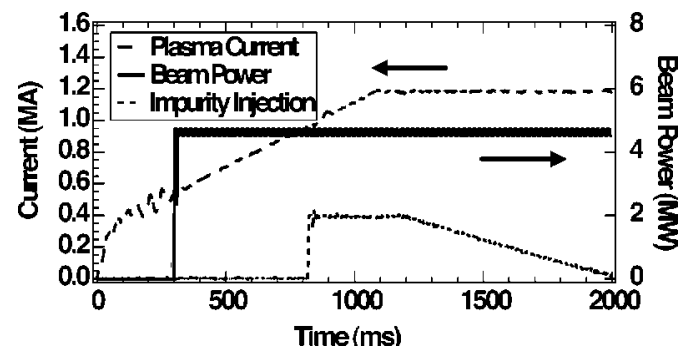

FIG. 1. Operational parameters $\left(I_{p}, P_{\text {beam }}\right.$ and gas injection) for the $L$-mode upper-biased double null divertor plasmas performed for these impurityinjection experiments. The early beam promotes a negative central magnetic shear profile.

edge regions of DIII-D with various diagnostics exhibit a dramatic reduction in turbulent density fluctuations as a result of impurity injection. Gyrokinetic simulations of these plasmas show qualitative agreement by exhibiting a reduction in linear growth rates and nonlinear saturated turbulence levels. These global and local experimental observations and preliminary theoretical understanding point to an operational mode that simultaneously achieves good confinement, while maintaining a highly radiative $L$-mode edge, both attractive features for a fusion reactor.

\section{DIII-D IMPURITY INJECTION EXPERIMENTS}

The discharges studied here have an $L$-mode edge, upper-biased double null divertor configuration, negative central magnetic shear (NCS) $q$ profile, a plasma current of 1.2 MA, and toroidal field of 1.6 T. Neutral beam power (4.5 MW) is injected starting at $0.3 \mathrm{~s}$ to heat electrons and thereby slow flux penetration to produce the NCS $q$ profile. This is turn inhibits sawteeth and various magnetohydrodynamic (MHD) modes, and may facilitate the formation of internal transport barriers. ${ }^{10}$ To allow more detailed study of the transport and turbulence dynamics, the beam power was relatively low to avoid higher $\beta$ discharges more prone to MHD behavior. Impurities were puffed in at various times, ranging from 0.8 to $1.2 \mathrm{~s}$. Noble gases (neon, argon, krypton) were puffed for these experiments due to their high recycling and lack of chemical reactivity with the graphite walls; there was no measurable buildup of impurity between shots. Impurity-injected discharges were compared to reference discharges with identical operational parameters, except that no impurity gas was puffed, providing a baseline from which to compare global parameters, profiles and fluctuation dynamics. The discharge evolution is shown in Fig. 1. For the discharges discussed next, neon was puffed at an initial rate of 2.0 Torr $\ell / \mathrm{s}$ starting at $0.8 \mathrm{~s}$ and was continuously injected, but with a decreasing flow rate that resulted in a peak neon density near $2 \%$ of the electron density.

Several global parameters from a neon-injected discharge are compared to those from the associated reference discharge in Fig. 2. We show the evolution of a neoninjected discharge in Fig. 2, rather than a discharge with argon or krypton injection, since neon exhibited the most dramatic confinement improvement in these experiments on DIII-D. Prior to the neon gas injection, the discharges are

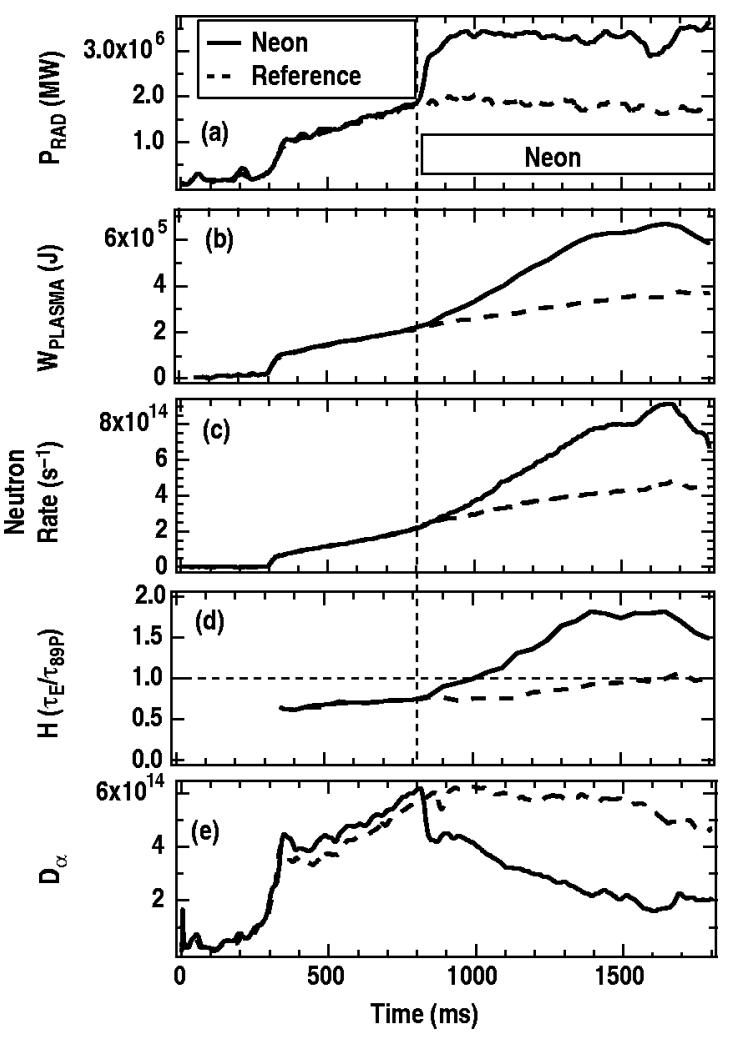

FIG. 2. Comparison of global confinement parameters for a neon-injected discharge (solid lines) and a reference discharge with no injected impurity (dashed lines): (a) radiated power, (b) plasma stored energy, (c) neutron rate, (d) energy confinement time normalized to $\tau_{89 P}$, and (e) $D_{\alpha}$ recycling emission.

nearly identical. Within $0.2 \mathrm{~s}$ of the neon injection, the radiated power increases from approximately $2 \mathrm{MW}$ to nearly $3.5 \mathrm{MW}$, radiating nearly $75 \%$ of the total input power. Coincident with the neon injection is an increase in the stored energy, energy confinement time, and neutron emission, all nearly doubling in magnitude by $1.6 \mathrm{~s}$, while there is a rapid reduction in the edge deuterium recycling $D_{\alpha}$ light. The energy confinement, defined here as

$$
\tau_{E}=W_{\text {PLASMA }} /\left(P_{\text {INPUT }}-d W / d t\right),
$$

with $W_{\text {plasma }}$ the stored energy, $P_{\text {input }}$ the total input power, and $d W / d t$ the rate of change in stored energy, nearly doubles, despite the increase in the radiative loses in the electron channel arising from the edge region. Also, the neutron rate is observed to increase rapidly, despite the modest dilution of the fuel ions from the impurity puff. $n_{\text {deuteron }} / n_{e}$ is 0.9 in the reference discharge and is reduced to 0.7 in the neon discharge at $1.6 \mathrm{~s}$ as a result of an increase in $n_{e} ; n_{\text {deuteron }}$ is nearly the same in both discharges. Shortly after $1.65 \mathrm{~s}$, the discharge performance begins to roll over due to the onset of $3 / 2$ neoclassical tearing mode activity. The MHD appears to be triggered by a sawtooth that coincides with the appearance of a $q=1$ surface in the plasma.

The neon injection broadens plasma profiles and increases central values. Profiles of ion temperature, toroidal rotation velocity, electron temperature, and electron density are compared with those of the reference discharge in Fig. 3 at $1.6 \mathrm{~s}$, near the peak performance in the neon-injected shot. 

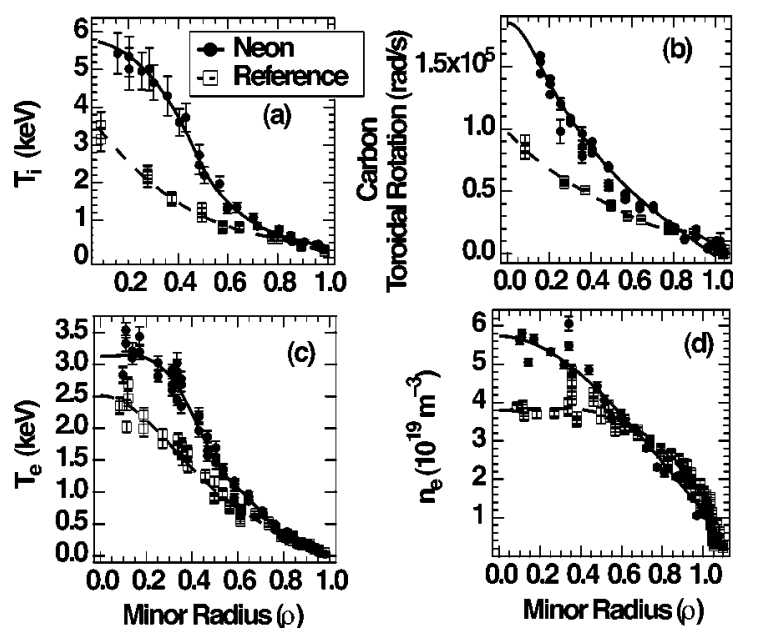

FIG. 3. Temperature and density profiles compared for neon (solid lines) and reference discharges (dashed lines) at $t=1.6 \mathrm{~s}$ : (a) ion temperature, (b) toroidal rotation, (c) electron temperature, and (d) electron density.

The ion temperature $T_{i}$ and carbon toroidal rotation rate $\Omega_{p}$ are seen to have much broader profiles and significantly higher central values. The central values of both $T_{i}$ and $\Omega_{p}$ nearly doubled, with changes occurring largely inside of $\rho$ $=0.8$. The electron temperature profile likewise exhibits a broadening and increase in central value, though not as dramatic a change as the ion temperature profile. The electron density profile exhibits two changes: a roughly $50 \%$ increase in core value, while the edge density drops modestly. A similar discharge in which the neon puff was replaced by a deuterium puff, providing a comparable electron source rate, did not exhibit an increase in peakedness so this result is not attributed to the electron source from neon.

The ion heat diffusivity profile shows a dramatic reduction across much of the profile, with $\chi_{i}$ reduced to near neoclassical levels. Ion and electron energy and momentum transport were calculated with the TRANSP kinetic analysis $\operatorname{code}^{11}$ using the measured plasma profiles. Profiles of the ion thermal diffusivity $\chi_{i}$ and electron diffusivity $\chi_{e}$ at $t$ $=1.16 \mathrm{~s}$, as well as time evolutions of these parameters at a $\rho=0.65$, are shown in Fig. 4. Electron heat diffusivity also shows a modest reduction in the outer regions of the plasma. The time history of $\chi_{i}$ exhibits a clear and rapid reduction in response to the neon gas puff. Outside of $\rho=0.7$, the uncer-
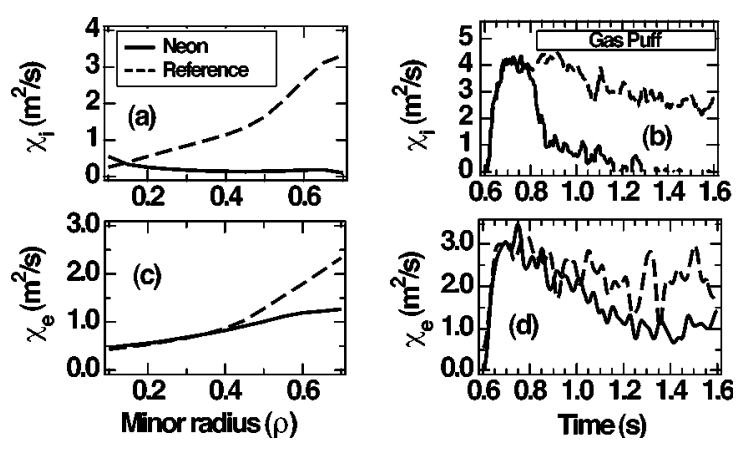

FIG. 4. Profiles of calculated transport coefficients obtained from TRANSP at $t=1.16 \mathrm{~s}$ and time histories at $\rho=0.65$ : (a), (b) $\chi_{I}$, (c), (d) $\chi_{e}$. tainty in the transport analysis becomes greater due to a number of factors including radiation and neutral effects, and thus the results are only shown out to $\rho=0.7$. The neon injected discharges here do not exhibit strong, spatially localized internal transport barriers, but rather a broad region of improved confinement that extends over much of the plasma minor radius, well outside of $\rho_{q, \min }$ which has been correlated with the radius of an internal transport barrier in past experiments. The large reduction in what is initially largely anomalous transport in the neon-injected discharges suggests an effect of impurity injection on turbulence, the mechanism believed to be driving the anomalous transport.

\section{TURBULENCE MEASUREMENTS AND CHARACTERIZATION}

A central goal of the DIII-D impurity injection experiments was to determine the underlying physical mechanism that gives rise to the transport reduction. It appears that suppression of turbulence by the impurity is at least partially, if not largely responsible for the confinement improvement and transport reduction. Turbulence is believed to drive anomalous transport and the two have been correlated in various studies. ${ }^{1,12}$ Detailed measurements of fluctuations arising from turbulence were obtained over a range of spatial locations and wave numbers with several diagnostics: beam emission spectroscopy (BES) ${ }_{15}^{13}$ far infrared scattering, ${ }^{14}$ and edge Langmuir probe arrays. ${ }^{15}$

BES measures localized density fluctuations in the wave number range $0 \leqslant k<3 \mathrm{~cm}^{-1}$ by observing the intensity of the Doppler-shifted $D_{\alpha}\left(\lambda_{0}=656.1 \mathrm{~nm}\right)$ light emission that arises from collisional excitation of the neutral beam atoms by background electrons and ions. Measurements are obtained with a poloidal and radial resolution of about $1 \mathrm{~cm}$, and are digitized at $1 \mathrm{MHz}$. The resulting light emission, and fluctuations therein, are directly related to the local density and density fluctuations through atomic physics. ${ }^{16}$ Measurements of density fluctuations from the BES diagnostic are compared for a neon-injected and reference discharge in Fig. 5 . BES spectra of the density fluctuations were obtained over the time interval $0.7-1.2 \mathrm{~s}$ in these discharges. The broadband density fluctuation spectra, obtained at a normalized minor radius of $\rho=0.7$, are compared before and after neon injection. These lab-frame measurements exhibit density fluctuations up to near $200 \mathrm{kHz}$ at $0.7-0.8 \mathrm{~s}$ [Fig. 5(a)], and up to $300 \mathrm{kHz}$ at $1.1-1.2 \mathrm{~s}$ [Fig. 5(b)]. The lab-frame frequency is determined by $\omega_{\text {lab }}=\omega_{\text {plasma }}+k_{\perp} v_{E \times B}$, with the Doppler shift term $\left(k_{\perp} v_{E \times B}\right)$ dominating the frequency as is typical for these plasmas. Therefore, the wave number is essentially proportional to frequency $\left(\omega_{\text {lab }} \approx k_{\perp} v_{E \times B}\right)$. The drift velocity $v_{E \times B}$ is measured directly with BES by applying time-delay correlation analysis between poloidally separated channels with $v=\Delta z / \Delta t{ }^{17}$ The approximate wave number scale, in units of $k_{\perp} \rho_{s}$, is shown on the upper axis. The drift velocity is slowly changing during the $0.1 \mathrm{~s}$ measurement interval so the average is taken to relate frequency to wave number. At the later time [Fig. 5(b)], the drift velocity has increased, broadening the frequency spectrum and 

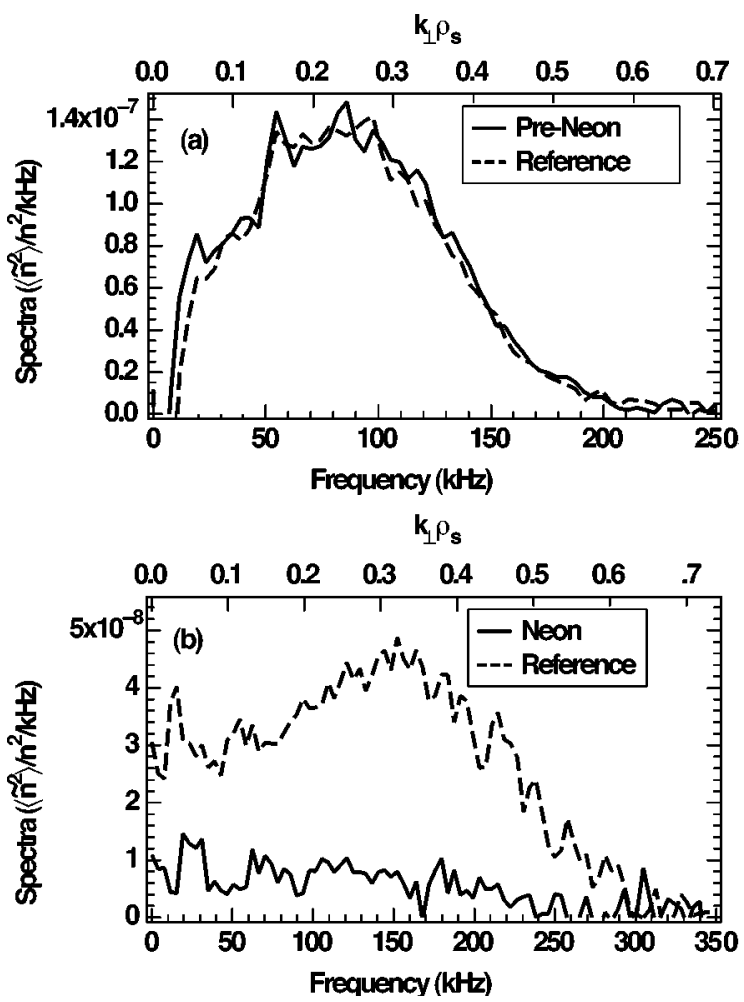

FIG. 5. Density fluctuation spectra measured with BES at $\rho=0.7$ at: (a) $t$ $=0.7-0.8 \mathrm{~s}$, prior to neon injection, and (b) $t=1.1-1.2 \mathrm{~s}, 0.3-0.4 \mathrm{~s}$ after start of neon injection showing substantial reduction in turbulence.

correspondingly changing the frequency to wave number mapping.

The fluctuation spectra from the neon and reference discharge are nearly identical prior to neon injection [Fig. 5(a)], as expected since the discharges are nearly identical at this point. At the later time interval [Fig. 5(b)], 300-400 ms after the neon injection, the fluctuation spectrum in the neon discharge is seen to be dramatically reduced in power relative to the reference discharge. This reduction in measured density fluctuations, and the associated anomalous transport, is believed to be at least partially, if not largely, responsible for the improvements in confinement that are observed in these discharges. The measured reduction in ion transport, which is believed to result from long wavelength turbulence $\left(k_{\perp} \rho_{s}<1\right)$, is consistent with this large suppression of fluctuations.

Temporally resolved measurements of the fluctuations from BES at $\rho=0.7$, shown in Fig. 6, exhibit two distinct time scales in the turbulence suppression dynamics in the neon-injected discharge. First, within $10 \mathrm{~ms}$ of the neon injection, there is a rapid drop of roughly $20 \%$ in the fluctuation amplitude. Second, over the next $400 \mathrm{~ms}$, there is a gradual reduction in the fluctuation amplitude that approaches the lower limit of detectability toward the end of the time window under examination. The early rapid drop occurs on a time scale that is fast compared to changes in the plasma profiles. One change that might occur on this time scale is that neon ions have transported rapidly through the edge $L$-mode region and reached the observation radius. Thus it appears that the presence of the neon alone may be

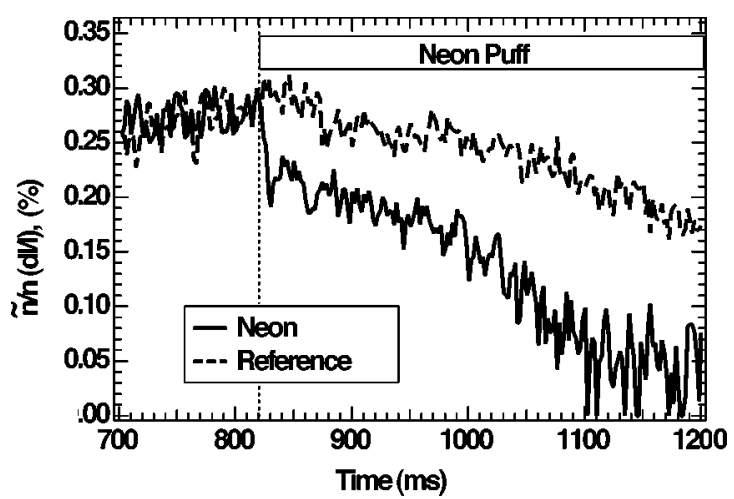

FIG. 6. Temporal evolution of density fluctuations, measured with BES at $\rho=0.7$, showing two characteristic time scales: prompt $20 \%$ drop in fluctuations within $10 \mathrm{~ms}$, followed by gradual reduction over 200-300 ms.

enough to initiate the turbulence suppression process. The second, longer time scale of $200-300 \mathrm{~ms}$ is of similar order to the time scale over which significant changes in the plasma profiles are taking place such as temperature increase and broadening, density peaking, and toroidal rotation increase.

The radial profile of density fluctuations was measured over the range $\rho=0.7-1.0$ and is shown Fig. 7 for $t$ $=1.1-1.2 \mathrm{~s}$. The large amplitude fluctuations near the edge of the plasma $(0.85 \leqslant \rho \leqslant 1.0)$ do not change significantly between the neon-injected and reference discharge. At a radius near $\rho=0.85$, the fluctuation amplitudes begin to diverge and by $\rho=0.7$, there is a significant reduction in the neon discharge. This range corresponds closely with the spatial region over which the ion temperature profile begins to increase [Fig. 3(a)], suggesting a correlation between the two.

The decrease in long wavelength density fluctuations has been observed with far infrared scattering (FIR). The FIR measurements were obtained at $2 \mathrm{~cm}^{-1}$, and are integrated over much of the plasma volume. The frequency of the fluctuations is dominated by the $E \times B$ Doppler shift of these long wavelength modes, as with the BES frequency spectra. Since the radial electric field has been determined as a func-

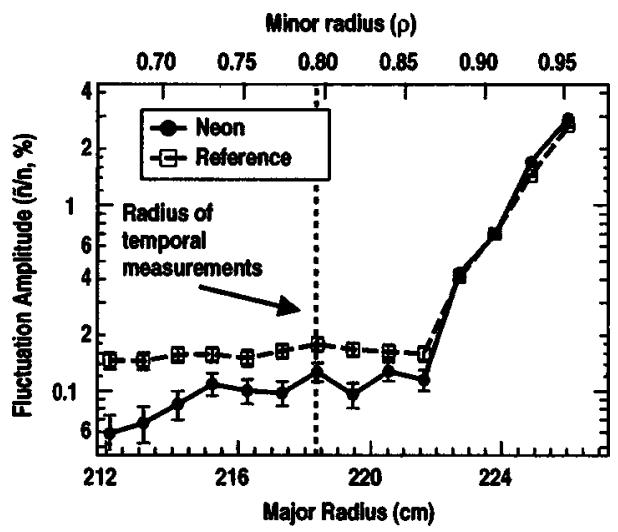

FIG. 7. Radial profile of density fluctuation amplitude measured with BES over radial range $0.7 \leqslant \rho \leqslant 1.0$ in neon and reference discharges showing larger amplitude reduction deeper in the core from neon, and little change outside $\rho=0.85$. 

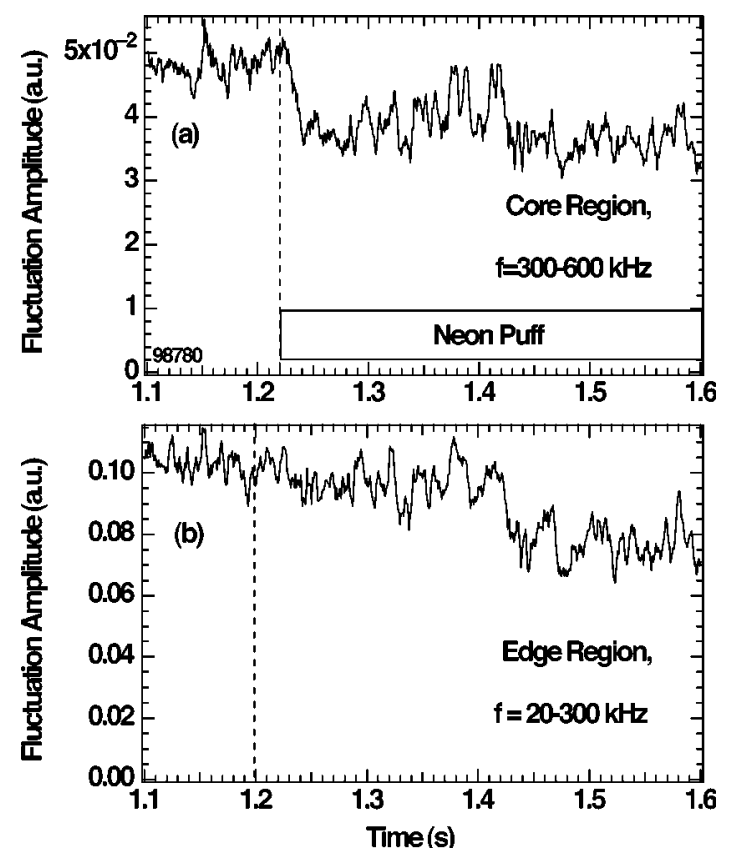

FIG. 8. Density fluctuation amplitudes measured with FIR in similar discharges to those discussed earlier, with neon puff at $1.22 \mathrm{~s}$, integrated over two frequency ranges: (a) 300-600 kHz, deeper in core, showing rapid reduction at neon injection time and (b) $20-100 \mathrm{kHz}$, reflecting edge fluctuation behavior.

tion of minor radius with charge exchange recombination spectroscopy, the $E \times B$ rotation velocity and associated Doppler shift can be determined, and thus frequency ranges can be correlated with spatial regions. ${ }^{18}$ Using this technique, edge and core fluctuation amplitudes have been inferred from the measured FIR spectrum in a different but similar neon-injected discharge, shown in Fig. 8. Figure 8(a) shows the core response to neon injection, integrated over a frequency range of $300-600 \mathrm{kHz}$. A fast drop in fluctuation amplitude occurs within about $10-20 \mathrm{~ms}$ of the neon injection, as was seen with BES (Fig. 6), while the edge fluctuation amplitude [Fig. 8(b),] integrated over $20-300 \mathrm{kHz}$ shows little change, again consistent with the BES profile measurements in Fig. 7.

Langmuir probe measurements demonstrated that although the amplitude of the edge density fluctuations does not change significantly with the injection of neon, the turbulent particle flux is reduced. Edge probes were inserted up to $2 \mathrm{~cm}$ inside the separatrix near the outer midplane to measure density and electrostatic potential fluctuations, their phase relationship, and thereby the turbulent driven particle flux. The electrostatic turbulent particle flux as a function of major radius is compared for a similar set of neon and deuterium-injected discharges in Fig. 9. The particle flux is seen to be significantly lower in the neon-injected discharge. The difference between the neon and reference discharges is attributed mostly to a change in the electrostatic fluctuation level, rather than the density fluctuation level. The particle flux is determined as $\Gamma_{r}=\left\langle\widetilde{n}\left(\widetilde{E}_{\theta} / B\right)\right\rangle$ and so it is the amplitude and phase relation between density and electrostatic potential that determines net turbulent-driven particle flux.

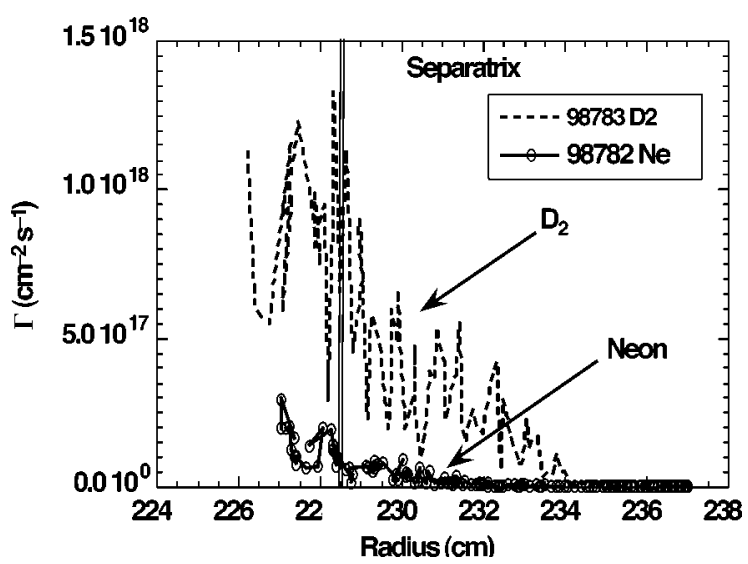

FIG. 9. Turbulent particle flux measurements obtained with the Langmuir probe array in the edge and scrape-off-layer region in a neon-injected discharge as well as a deuterium-puff discharge showing reduced edge particle flux with the neon injection.

These measurements of reduced flux are consistent with the decrease in edge recycling, seen in Fig. 2(e).

\section{DEPENDENCE ON IMPURITY QUANTITY AND ATOMIC NUMBER}

The confinement improves and measured density fluctuations decrease as the quantity of impurity injected is increased. A series of discharges were performed with the quantity of impurity gas (neon) injected varied to determine this dependence. The fluctuation spectra obtained with BES, again at $\rho=0.7$, are shown in Fig. 10(a) and the associated stored energy for these discharges is shown in Fig. 10(b). As the injected neon flow rate was increased from zero (reference), to 0.75 to $2.0 \mathrm{~T} \ell / \mathrm{s}$, the density fluctuation spectra decreased monotonically. The stored energy, correspondingly, increased monotonically with increasing neon. The neutron rate (not shown) likewise increases as stored energy increases. At the higher quantity of neon, approximately $75 \%$ of the input power is radiated away. More neon would

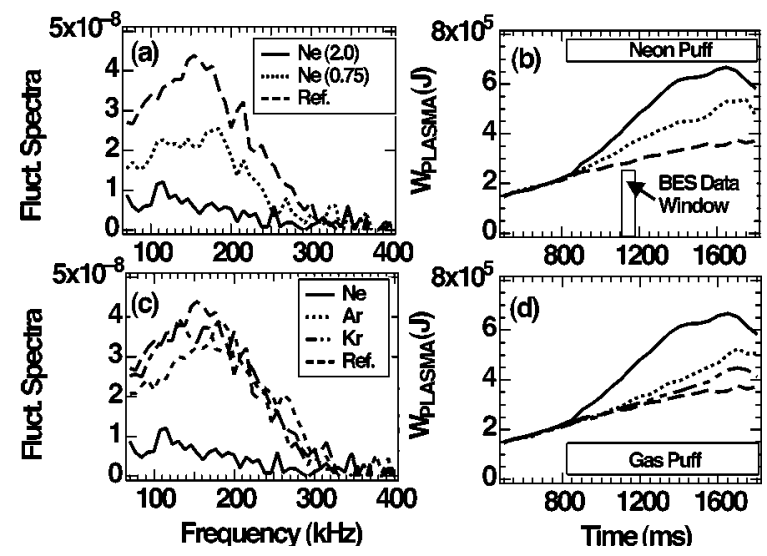

FIG. 10. Effects of varying impurity quantity and atomic number on confinement improvement: (a) BES fluctuation spectra at $\rho=0.7$ at $t$ $=1.1-1.2 \mathrm{~s}$, with three neon injection quantities: $0,0.75$ and 2.0 Torr $\ell / \mathrm{s}$, (b) stored energy in the same three discharges, (c) BES fluctuation spectra in four discharges, one each with neon, argon, krypton, and no impurity injection, and (d) associated stored energy response. 
likely result in a radiative collapse. A similar scan was performed with argon with qualitatively similar results: the more argon injected, the lower the fluctuation level and the higher the stored energy and neutron rates.

The atomic number dependence of the confinement improvement was examined by injecting argon $(Z=18)$ and krypton $(Z=36)$ as well as neon $(Z=10)$ into otherwise similar discharges. Neon exhibited the most dramatic improvement, although the other gases caused measurable confinement increases. In all three cases, the radiation level was approximately $75 \%$ of the injected power. For neon, the density of the fully stripped charge state, $\mathrm{Ne}^{+10}$, is directly measured with charge exchange recombination spectroscopy. ${ }^{19}$ The fully stripped ion dominates the neon density over much of the profile. Appreciable quantities of partially ionized states, mainly $\mathrm{Ne}^{+8}$ and $\mathrm{Ne}^{+9}$, exist only near the edge. The core $Z_{\text {eff }}$ was measured to be near 3.0 in these discharges. It was difficult to directly measure the total impurity quantity for argon and krypton since the charge state distribution consists of many partially ionized states, one of which is measured in the case of argon, and none for krypton. Thus it is not known how much dilution occurred with these higher $Z$ impurities. The qualitative result from this scan was that all three impurities exhibit some degree of turbulence suppression and confinement enhancement, shown in Figs. 10(c) and 10(d). Neon exhibited the most remarkable improvement and krypton the least, indicating an inverse $Z$ dependence to the confinement improvement. These results contrast with those obtained during experiments on TFTR, ${ }^{4}$ which showed the most significant improvement in confinement with krypton and xenon $(Z=54)$, as opposed to neon and argon. The TFTR experiments were performed at significantly higher temperatures which may account for the observed differences.

To further elucidate the DIII-D results, a discharge was performed in which the impurity gas puff was replaced with a deuterium gas puff to determine whether the density rise alone could cause the confinement increase. The deuterium gas puff closely reproduced the line-integrated density rise measured with the neon gas puff. The stored energy and density fluctuations, however, showed no measurable change relative to the reference discharge (with no extra deuterium or impurity puff), indicating that the confinement improvement specifically results from the neon (or argon or krypton) influx into the discharge and not simply a density rise.

\section{GYROKINETIC SIMULATIONS}

Turbulence in magnetically confined plasmas is driven by radial temperature and density gradients. Simulations have shown that long-wavelength turbulence, predicted to dominate anomalous ion transport, is largely driven by a combination of ion temperature gradient (ITG) modes and trapped-electron modes. ${ }^{20}$ Various studies have shown that impurities can affect the stability of various microinstabilities that are believed to give rise to turbulence and anomalous transport. ${ }^{21-25} \mathrm{~A}$ general result observed is that peaked impurity density profiles tend to stabilize ITG modes as a result of one of several mechanisms: dilution of the main

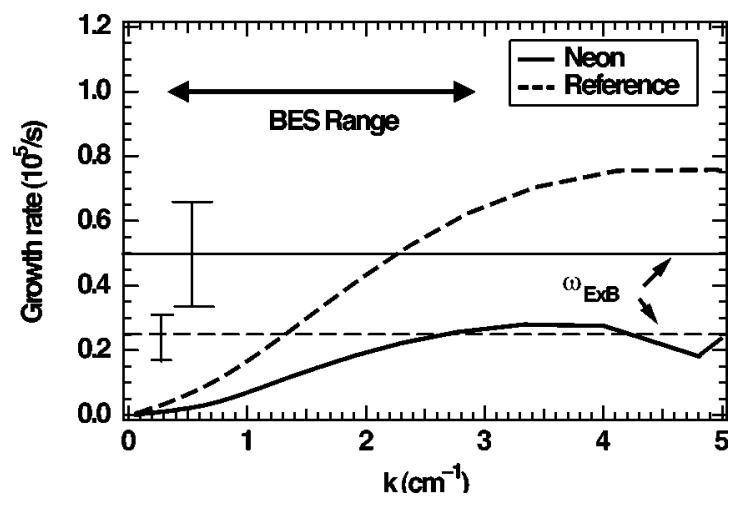

FIG. 11. Comparison of linear growth rates $\gamma_{\text {lin }}$ (curves) and $\omega_{E \times B}$ shearing rates (horizontal lines) in a neon-injected and reference discharge at $\rho$ $=0.7$ and $t=1.16 \mathrm{~s}$ showing that in the reference discharge $\gamma_{\text {lin }}>\omega_{E \times B}$, while in the neon-injected discharge $\gamma_{\text {lin }}<\omega_{E \times B}$ consistent with the confinement improvements.

fuel ions, direct mode stabilization by impurity ions, and temperature gradient changes resulting from enhanced localized radiation. In addition, $E \times B$ shearing affects the stabilization of microturbulence. In the $E \times B$ shearing model, ${ }^{26,27}$ turbulence is suppressed as the $\omega_{E \times B}$ shearing rate ${ }^{28}$ exceeds the linear growth rate of the most unstable modes, $\omega_{E \times B}>\gamma_{\text {lin, } \max }$, because of eddy shearing and nonlinear decorrelation. Therefore, we have examined the $\omega_{E \times B}$ shearing rates and the linear growth rates for these plasmas.

In the discharge with neon injection, the $\omega_{E \times B}$ shearing rate significantly exceeds the linear growth rate of the turbulence, consistent with the above paradigm and the measured reduction in density fluctuations. Linear growth rates were calculated with the gyrokinetic simulation (GKS) $\operatorname{code}^{29}$ using the measured density and temperature profiles. Contributions from ITG, trapped-electron, and electromagnetic modes are included. The $\omega_{E \times B}$ shearing rate is calculated from measurements of radial electric field obtained from radial force balance of the intrinsic carbon ions. These calculations are obtained with charge exchange recombination spectroscopy measurements of toroidal and poloidal rotation and pressure gradient. The resulting growth rates and shearing rates at $\rho=0.7$ and $t=1160 \mathrm{~ms}$, the location and time of the BES spectra presented in Fig. 5, are shown in Fig. 11. Two changes occur in the neon discharge relative to the reference discharge. One, the growth rate in the long wavelength region observed by BES is reduced by a factor of 2 or more. In addition, the shearing rate has increased and unlike the reference discharge, is now larger than the growth rate, thus achieving the conditions predicted to stabilize turbulence. These results show a clear, though qualitative, consistency with the BES spectra.

Predictions of fully saturated turbulence levels have been calculated with a nonlinear three-dimensional gyrokinetic particle simulation code ${ }^{30}$ again using the measured plasma profiles in a pair of discharges that are very similar to those discussed earlier. This nonlinear code incorporates fully kinetic ions and adiabatic electrons, and thus ITG modes are included in this simulation but no trapped-electron effects are considered. A discharge in which neon was injected at $1.22 \mathrm{~s}$ has been examined. The simulation was per- 


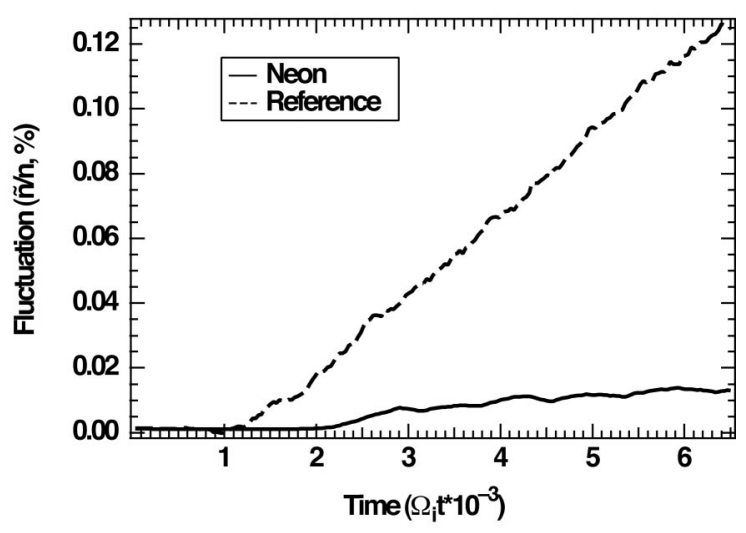

FIG. 12. Nonlinear gyrokinetic simulation of neon-injected and reference discharge showing the reduction in both the growth rate and nonlinear saturated level of density fluctuations in the neon-injected discharge at $t$ $=1.26 \mathrm{~s}, 40 \mathrm{~ms}$ after the neon injection in these discharges, at $\rho=0.7$.

formed at $t=1.26 \mathrm{~s}, 40 \mathrm{~ms}$ after the neon injection. This time was chosen since enough time has elapsed to allow the neon to penetrate into the plasma, and yet the profiles have just begun to change in response to the impurity injection. The simulation is allowed to evolve starting from a stationary equilibrium to examine how the turbulence develops. The time-evolved normalized density fluctuation levels, $\widetilde{n} / n$, [at $\rho=0.7]$ for a neon-injected and reference discharge are compared in Fig. 12. The time step used is the inverse of the ion gyrofrequency $\left(1 / \Omega_{i}\right)$ and is thus rapid compared to the evolution of the plasma. Two effects are observed. First, the linear growth rate is seen to be reduced in the neon-injected discharge, consistent with the growth rates calculated by the GKS code (Fig. 11). Second, the fully saturated (quasisteady state) amplitude is reduced. Globally, the turbulent electrostatic fluctuation energy saturates in these nonlinear simulations. Locally, however, the density fluctuations [at $\rho=0.7$ ] in the reference discharge are seen to not reach a steady state over the time window under examination. It is nevertheless clear that the equilibrium saturated level in the reference discharge will significantly exceed that of the neon-injected discharge. These results are again consistent with the fluctuation measurements and provide a framework from which to begin performing direct, quantitative comparisons between turbulence simulations and experimental fluctuation measurements.

\section{SUMMARY}

Injection of an impurity gas puff into an $L$-mode negative central shear discharge has been shown to significantly improve the global confinement parameters with the stored energy, neutron rate and energy confinement time nearly doubling compared to a reference discharge with identical control parameters, but no impurity puff. These improvements are correlated with a reduction in ion and electron transport, with ion energy transport being reduced to near neoclassical levels. The ion and electron temperature profiles exhibit increased central values and broadened profiles, while the density profile becomes significantly more peaked. Density fluctuation measurements obtained with BES and
FIR show that turbulence is dramatically reduced in the core in response to the impurity injection, while Langmuir probe measurements show reduced edge particle flux. This suppression of turbulence and the associated anomalous transport appear to be at least partially, if not largely, responsible for the confinement improvements. Gyrokinetic simulations of these plasmas using the measured density and temperature profiles indicate the linear growth rates and nonlinear saturated turbulence levels are reduced by the effect of impurity ions on the stability of ion temperature gradient modes. This reduction results from a combination of direct effects of the impurity ions on mode stability as well as indirect effects from profile changes resulting from the radiation. The reduced momentum transport results in higher toroidal rotation and resulting $E \times B$ shear that likely contributes to the turbulence suppression. Thus it appears that impurity-induced reduction of microinstability growth rates and increased $E \times B$ shear are acting synergistically to improve global confinement. In addition, these plasmas exhibit enhanced mantle and divertor radiation levels that more evenly disperse energy flux over the first wall, and thus reduce heat flux to divertor plates.

\section{ACKNOWLEDGMENTS}

The authors appreciate the support of the DIII-D technical and scientific staff for assisting in the performance and data analysis for these experiments. This research was performed under U.S. Department of Energy Contract Nos. DEFG03-99ER54463, DE-AC05-96OR22464, DE-AC0276CH03073, and W-7405-ENG-48, and Grant Nos. DEFG02-89ER53296, DE-FG03-95ER54294, DE-FG0386ER53266, and DE-FG02-91ER54109.

${ }^{1}$ B. A. Carreras, IEEE Trans. Plasma Sci. 25, 1281 (1997).

${ }^{2}$ E. A. Lazarus, J. D. Bell, C. E. Bush et al., J. Nucl. Mater. 121, 61 (1984).

${ }^{3}$ A. M. Messiaen, J. Ongena, U. Samm et al., Phys. Rev. Lett. 77, 2487 (1996).

${ }^{4}$ K. W. Hill, S. D. Scott, M. Bell et al., Phys. Plasmas 6, 877 (1999).

${ }^{5}$ D. Ernst et al., 17th IAEA Fusion Energy Conference, Yokohama, Japan, IAEA-F1-CN-69/EXP1/14 (International Atomic Energy Agency, Vienna, 1998).

${ }^{6}$ G. R. McKee, K. H. Burrell, R. J. Fonck et al., Phys. Rev. Lett. 84, 1922 (2000).

${ }^{7}$ G. L. Jackson, M. Murakami, G. M. Staebler et al., J. Nucl. Mater. 266269, 380 (1999).

${ }^{8}$ O. Gruber, A. Kallenbach, M. Kaufmann et al., Phys. Rev. Lett. 74, 4217 (1995).

${ }^{9}$ J. Boedo, D. Gray, R. Conn et al., Nucl. Fusion 40, 209 (2000).

${ }^{10}$ B. W. Rice, T. S. Taylor, K. H. Burrell et al., Plasma Phys. Controlled Fusion 38, 869 (1996).

${ }^{11}$ R. J. Hawryluk et al., Physics Close to Thermonuclear Conditions, Varenna, Italy, 1979 (Commission of European Communities, Brussels, 1979).

${ }^{12}$ R. J. Fonck, G. Cosby, R. D. Durst et al., Phys. Rev. Lett. 70, 3736 (1993).

${ }^{13}$ G. R. McKee, R. A. Ashley, R. J. Fonck et al., Rev. Sci. Instrum. 70, 913 (1999).

${ }^{14}$ C. Rettig, S. Burns, R. Philipona, W. A. Peebles, and N. C. Luhmann, Rev. Sci. Instrum. 61, 3010 (1990).

${ }^{15}$ R. Moyer, K. H. Burrell, T. N. Carlstrom et al., Phys. Plasmas 2, 2397 (1995).

${ }^{16}$ T. A. Gianakon, R. Fonck, J. Callen et al., Rev. Sci. Instrum. 63, 4931 (1992)

${ }^{17}$ R. D. Durst, R. J. Fonck, G. Cosby, and H. Evensen, Rev. Sci. Instrum. 63, 4907 (1992). 
${ }^{18}$ C. Rettig, W. A. Peebles, E. J. Doyle, K. H. Burrell, C. M. Greenfield, G. M. Staebler, and B. W. Rice et al., Phys. Plasmas 4, 4009 (1997).

${ }^{19}$ D. F. Finkenthal, The measurement of absolute helium ion density profiles in the DIII-D tokamak using charge exchange recombination spectroscopy, Ph.D. thesis, University of California, Berkeley, 1994.

${ }^{20}$ W. Horton, Rev. Mod. Phys. 71, 735 (1999).

${ }^{21}$ R. Sydora, University of Alberta (personal communication, 1999).

${ }^{22}$ R. R. Dominguez and G. M. Staebler, Nucl. Fusion 33, 51 (1993).

${ }^{23}$ R. Paccagnella, F. Romanelli, and S. Briguglio, Nucl. Fusion 30, 545 (1990).
${ }^{24}$ R. R. Dominguez, Nucl. Fusion 31, 2063 (1991).

${ }^{25}$ M. Fröjdg, M. Liljeström, and H. Nordman, Nucl. Fusion 32, 419 (1992).

${ }^{26}$ K. H. Burrell, Phys. Plasmas 4, 1499 (1997).

${ }^{27}$ R. E. Waltz, R. L. Dewar, and X. Garbet, Phys. Plasmas 5, 1784 (1998).

${ }^{28}$ T. S. Hahm and K. H. Burrell, Phys. Plasmas 2, 1648 (1995).

${ }^{29}$ R. E. Waltz and R. L. Miller, Phys. Plasmas 6, 4265 (1999).

${ }^{30}$ R. D. Sydora, V. K. Decyk, and J. M. Dawson, Plasma Phys. Controlled Fusion 38, A281 (1996). 